\title{
ACREDITACIÓN INSTITUCIONAL EN CHILE: UNA OPCIÓN EMERGENTE
}

Gonzalo Zapata*

* Ex secretario técnico adjunto. Comisión Nacional de Acreditación de Pregrado (CNAP) 


\section{RESUMEN}

El artículo expone, en primer lugar, los fundamentos sobre los cuales se ha puesto en marcha la acreditación de instituciones de la educación superior, así como las razones más importantes por las que las instituciones se han sumado a esta iniciativa impulsada por la Comisión Nacional de Acreditación de Pregrado (CNAP). Luego, se examinan los objetivos, orientaciones metodológicas, criterios y etapas del proceso, para concluir con una valoración de la acreditación como mecanismo de aseguramiento de la calidad de las instituciones de educación superior.

\section{ABSTRACT}

The paper first presents the principles underlying accreditation for higher education institutions and also the main reasons why educational institutions have joined this initiative fostered by the National Commission for Undergraduate Accreditation (CNAP). It then goes on to examine the goals, methodological orientation and stages of the process, and it concludes with a valuation of accreditation as a quality assurance system for higher education institutions. 


\section{ACREDITACIÓN INSTITUCIONAL EN CHILE: UNA OPCIÓN EMERGENTE}

\section{Presentación}

En el contexto de un sistema de educación superior marcadamente desregulado, la acreditación ha surgido como una alternativa de control moderna, flexible, acorde a las características de nuestras instituciones, y de rango o clase internacional. No se trata de una iniciativa que pretenda enfrentar toda la problemática del área. Su finalidad es introducir una cultura de evaluación que permita asegurar el cumplimiento de estándares básicos y fomentar permanentemente la calidad de las instituciones, así como permitir que la sociedad tenga una visión más transparente de la oferta de educación superior. Puede considerarse como un dispositivo de regulación del que participan, articuladamente, las instituciones de educación superior, actores representantes del medio disciplinario y/o profesional, agencias externas responsables de la acreditación y también, de algún modo, el público general.

Hasta hace poco tiempo, nadie habría pensado que una iniciativa como la que describo más adelante podría obtener el interés manifiesto y activo de un número significativo de instituciones, las que hoy representan más del 79\% de la matrícula de todo el sistema nacional de educación superior ${ }^{1}$. En efecto, la actitud de las instituciones frente a este tipo de procesos ha sido, a mi juicio, simplemente notable. Aun

1 Cabe destacar que el número de instituciones adscritas al proceso de acreditación representa el $85 \%$ de la matrícula del sector de las instituciones autónomas del sistema, sector elegible para incorporarse al proceso. 
cuando prácticamente nadie se ha opuesto al interés público de asegurar calidad en la educación superior, el cómo y quién -o quiénesdebían hacerlo (no sólo a quién o quiénes les corresponde dirigir el proceso, sino también a qué instituciones está destinada la acreditación) eran cuestiones que, hasta hace muy poco, generaban bastante polémica.

En mi opinión, los más importantes eventos que motivaron a las instituciones a evaluar y acreditar calidad fueron:

- El explosivo desorden del sistema de educación superior, producido por la apertura de nuevos y diversos programas de formación, y de sedes a lo largo de todo el país.

- El interés de las instituciones que honestamente velan por la calidad por marcar la diferencia, en un contexto de oferta cada vez más competitiva y sin regulación.

- Asociado a lo anterior, el compromiso de las instituciones por mejorar la calidad a partir de un diagnóstico obtenido mediante la aplicación de metodologías apropiadas y participativas.

- La confianza en las personas y en el procedimiento de evaluación que se ha ido construyendo para evaluar y acreditar calidad.

- Finalmente, el hecho de que se trata de iniciativas internacionales, percibidas como una manera efectiva de obtener reconocimiento.

La opción metodológica adoptada por el Ministerio de Educación y la Comisión Nacional de Acreditación de Pregrado (CNAP) ${ }^{2}$ parece haber sido la correcta: los procesos de evaluación de la calidad se iniciaron de un modo experimental y abiertamente participativo, particularmente en lo referente al diseño de los procedimientos y criterios de evaluación, avanzándose así en la construcción colectiva de una experiencia ajustada a las características del sistema.

2 La CNAP es una entidad creada por decreto del Ministerio de Educación, con el objeto de diseñar y llevar a cabo procesos experimentales de acreditación. Inició sus actividades en 1999 y está integrada por 14 académicos de experiencia y trayectoria en el medio. Cuenta, además, con una secretaría técnica responsable de coordinar las acciones y llevar a cabo sus funciones. 
La incorporación de las instituciones a los procesos experimentales de acreditación fue paulatina y creciente. Las primeras generaron un efecto de demostración sobre el resto, manifestando su honesto compromiso con la calidad y, lo más importante, exhibiendo resultados útiles interna y externamente.

\section{La iniciativa de acreditación institucional}

La CNAP inició los procesos experimentales de acreditación en el ámbito de las carreras y programas debido, principalmente, al interés manifestado por algunas áreas profesionales y disciplinarias. Resultó un proceso eficiente para iniciar el aseguramiento y fomento de la calidad, por cuanto ubicó la evaluación en un nivel relativamente pequeño y manejable, donde es más fácil identificar fortalezas y debilidades y, por sobre todo, actuar sobre ellas para superar las deficiencias identificadas. Al mismo tiempo, concitó con facilidad el interés, participación y compromiso de la comunidad académica con la evaluación y sus resultados, permitiendo avanzar en el desarrollo de una "cultura de la evaluación" al interior de las instituciones.

Con el avance de los procesos de acreditación de las carreras resultó evidente que un sistema completo de aseguramiento de la calidad debía incluir también la de las propias instituciones. Hubo múltiples razones que fueron asentando la necesidad de avanzar en este desarrollo, entre las que destaca el hecho de que, frecuentemente, los problemas y las acciones identificados como consecuencia de la evaluación en las carreras o programas correspondían a decisiones institucionales más que a aspectos manejables desde una unidad académica responsable de administrar un currículo de formación.

Es importante indicar que el foco, metodología y resultados a los que conducen ambos tipos de acreditación son evidentemente distintos. Ninguno reemplaza o sustituye al otro, sino que se trata de procesos complementarios.

La acreditación institucional permite efectuar un análisis global, indispensable para poner en perspectiva los resultados de evaluaciones más específicas; si bien aporta información acerca del conjunto de la institución, y precisamente debido a la globalidad del análisis, no necesariamente da cuenta de la oferta específica de las carreras. 
Sólo a principios del año pasado la CNAP inició su primera experiencia de acreditación institucional, la que ha arrojado ya sus primeros resultados. Para ello, convocó a todas las instituciones autónomas del país a incorporarse a un proyecto piloto, cuyos objetivos fueron: dar garantía pública del grado en que las instituciones de educación superior cuentan con procesos eficaces para asegurar en cumplimiento de sus propósitos y mejorar los servicios ofrecidos, y promover en el seno de las instituciones la existencia de políticas y mecanismos permanentes de autorregulación de la calidad en las diferentes funciones institucionales y en los procesos conducentes a la obtención de logros y resultados esperados.

El diseño del proyecto ha resultado ser bastante innovador. En efecto, al establecer que la calidad sólo puede alcanzarse a través del compromiso activo de cada institución, resulta evidente que son los mecanismos internos de aseguramiento de la calidad los que pueden dar cuenta pública sobre dicho compromiso, lo que resulta especialmente interesante si la calidad se confronta con el crecimiento explosivo de la oferta de servicios de educación superior en el país.

Por ello, la acreditación institucional está planteada como una auditoría académica ${ }^{3}$, esto es, la evaluación de la capacidad institucional para la autorregulación, entendida como el conjunto de políticas, mecanismos, procedimientos y acciones destinados a determinar si la institución está efectivamente avanzando hacia el logro de sus propósitos y objetivos.

En este sentido, el foco de la evaluación se pone en los procesos mediante los cuales las instituciones aseguran el cumplimiento de estándares académicos y mejoran la calidad de los servicios que prestan. El centro del análisis no está puesto directamente en la calidad intrínseca de las instituciones, sino en la gestión de la calidad; en otras palabras, en la forma en que una institución evalúa su situación presente, la compara con sus propósitos y objetivos, y define acciones concretas para aproximarse a las condiciones deseadas.

3 Se entiende por auditoría académica al proceso de evaluación externa (a cargo de pares evaluadores), en el cual se revisa cuidadosamente cada uno de los mecanismos de autorregulación asociados a áreas particulares, su consistencia, desarrollo y resultados. 
Se trata de un proceso más flexible y simple y, por otra parte, menos costoso e interventor que la evaluación institucional directa. Uno de sus elementos claves es que resguarda de mejor manera la autonomía de las instituciones de educación superior, enfatizando la necesidad de mejorar la gestión.

\section{Orientaciones metodológicas}

Si el foco son las políticas y mecanismos de aseguramiento de la calidad de una institución en el contexto de su misión y propósitos institucionales, ello permite, entonces, que la evaluación se ajuste a distintos tipos de instituciones y respete la diversidad de misiones y propósitos institucionales. En efecto, podrán existir una multitud de maneras de asegurar calidad y de organizarse en virtud de los propósitos que persigue una determinada institución y su particular modo de ser; lo importante, en cualquier caso, es garantizar que lo haga a partir de mecanismos precisos de aseguramiento de la calidad, que deben ser aplicados sistemáticamente y exhibiendo resultados en consecuencia.

Con el objeto de resguardar estándares académicos mínimos, la acreditación institucional considera un "piso" que debe ser respetado y que tiene que ver con lo que usualmente se espera que considere una institución de educación superior en su misión y fines. Si bien el proyecto ha sido diseñado de modo tal que permite amplios grados de diversidad en cuanto a sus finalidades, no todo es aceptable. No lo es, por ejemplo, que los fines de una institución omitan que la formación técnica o profesional impartida sea de calidad y ajustada a los requerimientos propios del medio externo que se ha definido, o bien, que no se hagan cargo de las características de los alumnos que recibe, proporcionándoles una formación pertinente. Se trata de estándares básicos que resguardan que las instituciones efectivamente sean de nivel superior y que otorguen lo que, usualmente, la sociedad espera de ellas. Sobre dichos estándares, las instituciones pueden diferenciarse y definir sus propios proyectos académicos. A partir de dichos estándares, todo es admisible y a la vez exigible. En efecto, la misión y propósitos definidos son la medida de evaluación para cada institución.

Metodológicamente, el proyecto piloto de acreditación institucional fijó áreas en las que se desarrolla la evaluación de los 
mecanismos de aseguramiento de la calidad. Estableció como áreas mínimas de evaluación la gestión institucional y la docencia de pregrado, puesto que son esenciales para toda institución de educación superior y las más relevantes para el medio externo, particularmente para los estudiantes y sus familias: ¿cómo se organiza una institución para cumplir a cabalidad con sus metas y propósitos? Y ¿de qué forma administra su docencia de pregrado y asegura la calidad de la misma?

Además de las mínimas ya mencionadas, y con el fin de dar cuenta de la diversidad del sistema de educación superior, las instituciones pueden evaluarse en áreas adicionales, entre las que se cuentan: la investigación, la docencia de posgrado, la vinculación con el medio y la gestión de su infraestructura y equipamiento. Se ha estimado que estas áreas electivas permiten a las instituciones dar cuenta más fidedigna de su misión y propósitos institucionales cuando éstos son más complejos e incorporan funciones que van más allá de la formación de pregrado. Las áreas electivas se encuentran abiertas, con el objeto de que las instituciones puedan convenir con la Comisión él o los aspectos específicos adicionales que les resultan más apropiados de incorporar al proceso y les permiten perfilarse más acabadamente.

Para evaluar las áreas fue necesario definirlas operacionalmente, adoptando términos de referencia que establecen qué aspectos deben ser evaluados en el contexto de cada área. Por ejemplo, la evaluación del área de docencia de pregrado considera, entre otros aspectos, las políticas y mecanismos destinados a asegurar calidad en el diseño de los programas ofrecidos, la selección de los alumnos, los procesos académicos, el cuerpo académico asociado y los recursos involucrados ${ }^{4}$.

Todo este proceso tiene por objeto concluir, para cada una de las áreas seleccionadas, el grado en que la institución satisface los siguientes criterios:

- Cuenta con propósitos y fines institucionales apropiados y claros que orientan adecuadamente su desarrollo.

4 Los antecedentes relativos al procedimiento, criterios, áreas y términos de referencia de la evaluación, pueden ser consultados en el documento Proyecto Piloto de Acreditación Institucional, CNAP, disponible en la página web de la Comisión: www.cnap.cl 
- Cuenta con políticas y mecanismos formalmente establecidos para el aseguramiento de la calidad y el cumplimiento de sus propósitos institucionales.

- Presenta evidencias de que sus políticas y mecanismos de aseguramiento de la calidad se aplican sistemáticamente en los diversos niveles institucionales y de modo eficiente y eficaz.

- Presenta evidencias de resultados concordantes con los propósitos declarados y cautelados mediante las políticas y mecanismos de autorregulación.

- Demuestra que tiene capacidad para efectuar los ajustes y cambios necesarios para mejorar su calidad y avanzar consistentemente hacia el logro de sus propósitos declarados.

El juicio de acreditación final indica si una institución se ajusta a estos criterios, garantizando que cuenta con niveles mínimos de calidad y que vela por el cumplimiento adecuado de sus propósitos en las áreas que ha sometido a la evaluación.

La organización del proceso considera las siguientes etapas, usuales en este tipo de procedimientos:

- Ajuste a los estándares de calidad. En primer lugar, la institución garantiza que cumple con los estándares mínimos de calidad. El ajuste de una institución a estos estándares es un supuesto básico y constituye un prerrequisito o condición de elegibilidad para la acreditación institucional.

- Evaluación interna. Referida al análisis conducido por la propia institución sobre sus políticas y mecanismos destinados a asegurar la calidad de la gestión y la docencia de pregrado; también de cualquier área adicional que la institución haya decidido incorporar. Igualmente debe contemplar el análisis de los resultados de la aplicación de dichas políticas y mecanismos.

- Evaluación externa. Sobre la base del informe que sintetiza los resultados de la evaluación interna, un equipo de evaluadores externos visita la institución y efectúa una auditoría académica, destinada a determinar si la institución cuenta con políticas y mecanismos de autorregulación apropiados, y si el funcionamiento de éstos permite asegurar su calidad en las áreas definidas. 
- Decisión de acreditación. Pronunciamiento, por parte de la CNAP, de un juicio relativo a la existencia formal y a la aplicación eficaz de las políticas y mecanismos institucionales de aseguramiento de la calidad de las principales funciones institucionales.

\section{La evaluación interna}

Consiste en el proceso analítico destinado a identificar las prioridades, fortalezas y debilidades de la institución, relativas a los mecanismos institucionales que velan por el cumplimiento de la misión y propósitos institucionales.

Aún cuando la evaluación interna tiene muchas de las características de un proceso de autoevaluación no se ha querido utilizar dicha denominación, considerando que no necesariamente tiene sus características de participación amplia. Se trata, más bien, de un proceso conducido desde las principales instancias de toma de decisión, que consulta, eso sí, a los demás niveles de la institución.

La clave del proceso de evaluación interna es la identificación y posterior análisis de la aplicación de los mecanismos de aseguramiento de la calidad al interior de la institución y en sus diversos niveles. Este es un tema crítico e importante de dejar en claro; lo que se busca no es sólo que existan adecuadas políticas y mecanismos de aseguramiento de la calidad en determinadas áreas y procesos asociados, sino que, fundamentalmente, estos se apliquen rigurosamente y den los resultados esperados.

La evaluación debe analizar en detalle cómo se aplican dichas políticas en el nivel microorganizacional, es decir, cómo se asegura la calidad en el diseño de los programas, tanto en sus distintas facultades como en sus distintas sedes; cómo se operacionalizan las políticas de desarrollo académico definidas en el nivel central en cada una de las unidades académicas; cómo funcionan los mecanismos de apoyo a los estudiantes en las carreras de ingeniería, educación y ciencias; cómo responden los distintos servicios estudiantiles (laboratorios, biblioteca, talleres) al modelo pedagógico impulsado desde la vicerrectoría académica ${ }^{5}$.

5 El detalle de los aspectos objeto de la evaluación se encuentran en los términos de referencia definidos para cada una de las áreas consideradas en el proyecto piloto de acreditación. 
No debe sorprendernos que, por la naturaleza de las instituciones, existan efectivamente políticas y mecanismos de aseguramiento de la calidad con distinto nivel de formalidad y que, al ser aplicadas, no operen indefectiblemente del modo preestablecido o esperado. No debiera sorprendernos tampoco, que las instituciones cuenten con procedimientos formales que obedezcan más a construcciones burocráticas (o al modo como siempre se han hecho las cosas) que a mecanismos apropiados para el logro de los resultados esperados. La pregunta de fondo es si, efectivamente, las normas definidas y los procedimientos aplicados están alineados coherentemente para asegurar calidad en la práctica diaria de las unidades académicas.

Las instituciones cuentan con un instrumento de gran utilidad para perfeccionar el modo mediante el cual aseguran calidad, monitoreando su quehacer en los diversos niveles de operación; ha comenzado a difundirse como una herramienta estratégica que permite efectuar un diagnóstico útil y pertinente.

Las orientaciones metodológicas del proceso de evaluación consideran pasos prácticos a través de los cuales una institución debe auscultar cómo se aplican sus políticas y mecanismos de aseguramiento de la calidad, cuáles operan de modo correcto arrojando los resultados esperados y cuáles es preciso revisar y ajustar en el futuro ${ }^{6}$.

\section{La evaluación externa}

La selección y el desempeño de los pares evaluadores que participan en la etapa de evaluación externa son aspectos particularmente importantes en el proceso. Los pares verifican en terreno el cumplimiento de los criterios definidos y revisan que, efectivamente, las políticas y mecanismos definidos operen de modo correcto, asegurando calidad.

Fue necesario definir criterios muy claros para su selección. Las instituciones participantes propusieron formalmente nombres de posibles pares evaluadores; su selección se ajustó a criterios rigurosos, entre los que se cuentan su experiencia y trayectoria, el ejercicio de

6 Más antecedentes sobre el proceso de evaluación interna pueden encontrarse en la Guía de Evaluación Interna, documento preparado por la Secretaría Técnica de la CNAP y disponible en www.cnap.cl 
funciones de dirección académica y administrativa, su idoneidad disciplinaria y profesional y su reconocimiento en el medio como evaluadores experimentados e independientes.

Asimismo, fue necesario tener mucho cuidado al conformar los comités de pares; considerando el tamaño del medio nacional, fue indispensable definir y aplicar normas sobre incompatibilidades y conflicto de intereses.

Se propuso un conjunto de pares evaluadores a cada una de las instituciones, las que tuvieron el derecho a vetar a uno o más de los propuestos y a solicitar la revisión de la composición de todo el comité, todo lo cual se realizó de modo confidencial entre la Comisión y las instituciones participantes. La CNAP estaba especialmente interesada en que los pares evaluadores fueran aceptados por las instituciones para que sus juicios fueran asumidos de modo constructivo.

Cada comité estuvo integrado por cuatro a seis pares evaluadores, entre las que se contempló la participación de un académico extranjero de reconocido prestigio internacional. Esta decisión permitió, además de enriquecer la mirada y elementos de juicio de la evaluación, evitar, en parte, los posibles niveles de autorreferencia y clausura.

Debido a la novedad del enfoque metodológico, todos los evaluadores tuvieron que participar en una jornada de entrenamiento, en la que discutieron la lógica y enfoque definido, y se familiarizaron con los criterios, normas y materiales. La evaluación externa fue organizada por cada uno de los comités, quienes planificaron sus actividades con tiempo, contando para ello con los informes de evaluación presentados por las instituciones.

El desarrollo de estas primeras visitas de evaluación tuvo una duración de tres a cuatro días, dependiendo del tamaño de las instituciones y del número de áreas sometidas a la evaluación. Los comités, luego de las visitas en terreno, elaboraron sus informes de evaluación, los que fueron enviados por la CNAP a los rectores, quienes, por su parte, tuvieron la posibilidad de formular indicaciones o reparos a las conclusiones allí planteadas ${ }^{7}$.

7 Más antecedentes relativos al proceso de evaluación externa pueden encontrarse en el Manual de Pares Evaluadores, documento preparado por la Secretaría Técnica de la CNAP y disponible en www.cnap.cl 
Este tipo de ejercicios consume tiempo y genera ruido al interior de las instituciones. Sin embargo, es altamente enriquecedor y moviliza acciones concretas de fomento de la calidad. Sus beneficios son mayores cuando es conducido de modo honesto y abierto, y son claramente limitados cuando el ejercicio se realiza sólo en el plano formal, buscando exclusivamente cumplir con las disposiciones establecidas.

A modo de conclusión

Del total de 59 instituciones que participan en el proceso de acreditación institucional, la CNAP ha finalizado una primera etapa de evaluación acreditando a las primeras 13, que en su mayoría ya contaban con experiencias previas de evaluación.

El aprendizaje de este primer ciclo de acreditación fue relevante, no sólo desde el punto de vista operacional del proyecto -ya que se identificaron algunos aspectos necesarios de ser corregidos en futuras etapas-sino, fundamentalmente, considerando el impacto interno de la evaluación en las instituciones. De la lectura de los informes de evaluación, tanto internos como externos, se puede observar claramente cómo cada institución, a partir de sus propias características, identificó aspectos críticos en los que es preciso trabajar para elevar aún más los niveles de calidad.

Evidentemente queda aún mucho por aprender. Se trata de experiencias jóvenes que es preciso perfeccionar en el tiempo, monitorear su desempeño y fortalecer sus procedimientos de modo tal de incrementar su impacto. Tienen que ver con las preocupaciones más básicas de las instituciones de educación superior, que de uno u otro modo han venido enfrentando en el pasado, pero respecto de las cuales hoy cuentan con una nueva herramienta que les otorga la prioridad y urgencia requerida: cómo gestionar calidad en el cumplimiento de sus propósitos y objetivos institucionales, en un contexto crecientemente más complejo y competitivo.

Los ejercicios de acreditación institucional ponen el énfasis en la dinámica interna de las instituciones, insistiendo en que sean sus autoridades y académicos los que definan él o los modos mediante los cuales se garantiza y promueve calidad. Incorporan un proceso previo 
de definición de criterios que asegura la legitimidad, transparencia y validez del proceso. Ponen de relieve que la autonomía de las instituciones de educación superior es un aspecto esencial para el mejoramiento sostenido de la calidad, pero dejan en claro que la autonomía sólo se entiende y justifica en el marco de la responsabilidad con que las instituciones de educación superior asumen la labor que la sociedad espera de ellas. 